\title{
A Novel Method for Initial Rotor Position Estimation for IPM Synchronous Machine Drives
}

\author{
Hyunbae Kim, Student Member, IEEE, Kum-Kang Huh, Student Member, IEEE, Robert D. Lorenz, Fellow, IEEE, \\ and Thomas M. Jahns, Fellow, IEEE
}

\begin{abstract}
Novel methods for initial position estimation during startup are presented for interior permanent-magnet synchronous machine (IPMSM) drives. The magnet cavities in the IPMSM rotor create a sizable difference in the inductances of the two orthogonal rotor axes. This spatial saliency based on the rotor position makes it possible to use persistent, rotating or pulsating vector, carrier-frequency image tracking techniques to reliably identify and track the orientation of the $d$ and $q$ axes even when the rotor is at standstill. However, additional details in the saliency image must be used to identify the polarization of the magnets in order to distinguish the north and south poles. The magnet polarity is identified using magnetic saturation effects on the saliency image to uniquely identify the polarity being tracked. Carrier currents for both rotating and pulsating voltage carrier injection are derived by using IPM machine model including saturation and verified by measured carrier current components. Experimental results show that the proposed algorithms are capable of reliable and fast initial position estimation including the polarity at standstill.
\end{abstract}

Index Terms-Automotive applications, motion control, permanent-magnet (PM) motors, sensorless control.

\section{INTRODUCTION}

A classical method for initial position detection is to use Hall effect detectors to detect the permanent-magnet (PM) flux. The resolution of initial position is $180^{\circ}$ divided by number of Hall-effect detectors if the Hall-effect detector's signal conditioning has a binary output, i.e., is configured as a Hall-effect switch. For classical implementations using three Hall-effect switches, the error due to the limited angular resolution degrades starting torque. Additional resolution can be gained using an analog output Hall-effect sensor, at the expense of an additional $\mathrm{A} / \mathrm{D}$ converter for each sensor.

Paper IPCSD-04-035, presented at the 2003 Industry Applications Society Annual Meeting, Salt Lake City, UT, October 12-16, and approved for publication in the IEEE TRANSACTIONS ON INDUSTRY APPLICATIONS by the Industrial Drives Committee of the IEEE Industry Applications Society. Manuscript submitted for review August 1, 2003 and released for publication May 24, 2004. This work was supported by the Wisconsin Electric Machines and Power Electronics Consortium (WEMPEC) of the University of Wisconsin, Madison. The work made use of ERC Shared Facilities supported by the National Science Foundation (NSF) under AWARD EEC-9731677.

H. Kim was with the Department of Mechanical Engineering, University of Wisconsin, Madison, WI 53706 USA. He is now with the Living Appliances R\&D Center, Samsung Electronics, Suwon-si 442-742, Korea (e-mail: h24.kim@samsung.com).

K.-K. Huh and T. M. Jahns are with the Department of Electrical and Computer Engineering, University of Wisconsin, Madison, WI 53706 USA (e-mail: kumkanghuh@wisc.edu; jahns@engr.wisc.edu).

R. D. Lorenz is with the Departments of Mechanical Engineering and Electrical and Computer Engineering, University of Wisconsin, Madison, WI 53706 USA, (e-mail: lorenz@engr.wisc.edu).

Digital Object Identifier 10.1109/TIA.2004.834091
A critical aspect to modern drive applications is reliability. A key factor affecting the reliability of motor drives is the position sensor. The additional wiring and connections to sensors degrades reliability. A simple, low-cost approach to obtain a useful initial position without requiring a position sensor involves using dc current excitation to physically align the rotor to an initial position. However, with dc current excitation, the direction of rotor rotation is unpredictable, the rotor is aligned slowly and the mechanical system must be free to rotate. Such physical compromises are not suitable for many applications.

Over the last decade, several solutions have been proposed for both speed and position sensorless methods for the permanentmagnet synchronous machine (PMSM) [2]-[15]. Initial rotor position estimation for the PMSM has been an ongoing topic of research. Two basic methods for initial position estimation are pulse signal injection [2]-[6] or sinusoidal carrier-signal injection [7]-[15].

The pulse signal injection methods are often based on estimating the minimum inductance location using a calculated $d i / d t$ obtained during some form of iterative square wave voltage injection to arbitrary axes such as that in [2]-[6]. Such methods can be applied to both surface [2]-[4], [6] and interior PMSM (IPMSM) [5], [6]. A search algorithm for an optimum voltage vector that uses effective magnetic saturation without rotating the rotor was introduced in [4]. Magnetic axis without polarity was estimated via a method named "INFORM" (Indirect Flux detection by On-line Reactance Measurement) and the polarity was detected by finding minimum inductance on the estimated magnetic axis [6]. In these methods, initial position estimation accuracy can be affected by additional spatial harmonics such as saturation of the stator teeth.

Injection of a high-frequency, rotating [7]-[10], [15] and/or pulsating [11]-[15] excitation has also been widely used to estimate initial rotor position using either voltage [7]-[11], [14], [15] or current injection [12], [13]. In [8], the magnetic axis was identified from the current locus through rotating voltage injection and magnet polarity was detected by finding minimum inductance via square-wave voltage injection for an IPMSM. A rotating carrier voltage vector has also been injected into IPMSMs [7] and PMSMs [9], tracking the spatial saliency image without polarity detection. In [10], an experimentally determined magnitude-based current envelope was used to detect magnet polarity. In the carrier-signal injection methods, the location of the magnetic axis can be estimated using the carrier-signal current resulting from the interaction between the carrier-signal voltage and the spatial saliency. The magnet polarity should be simultaneously estimated using a polarity dependent saturation effect. A 
high-frequency, pulsating voltage [11], [14], [15] or current [12], [13], [15] vector in the estimated synchronous frame was injected into an IPM machine for the initial position. Saturation-dependent voltage reference oscillation of the current regulator near the peak current was observed and used for magnet polarity detection [12]. A first-order $d$-axis inductance model including saturation was introduced to detect magnet polarity [13] and flux linkage. The polarity-dependent $d$-axis magnetic saturation was modeled as a function of current by a second-order Taylor series [15]. The magnet polarity was determined from the second harmonic of the injected frequency [13]-[15]. An accurate saturation model is required to improve the convergence speed of the polarity estimation for carrier-signal injection methods.

This paper presents initial rotor position estimation techniques based on spatial saliency tracking with polarity detection via rotating or pulsating vector carrier voltage injection. Accurate IPMSM models, including saturation, for both carrier-signal injections are introduced. Compact signal processing of polarity detection is developed based on these models. The results are simple, robust techniques for quickly estimating the location and magnet polarity of an IPMSM.

\section{IPMSM MODEL INCLUDING MAGNETIC SATURATION FOR CARRIER-SIGNAL INJECTION}

The IPMSM machine model can be represented in scalar form using stator flux in the stationary frame as (1) and (2)

$$
\begin{gathered}
v_{d s}^{r}=r_{s} i_{d s}^{r}+p \lambda_{d s}^{r}-\omega_{r} \lambda_{q s}^{r} \\
v_{q s}^{r}=r_{s} i_{q s}^{r}+p \lambda_{q s}^{r}+\omega_{r} \lambda_{d s}^{r}
\end{gathered}
$$

where

$$
\begin{aligned}
& \lambda_{d s}^{r}=L_{d} i_{d s}^{r}+\lambda_{p m} \\
& \lambda_{q s}^{r}=L_{q} i_{q s}^{r} .
\end{aligned}
$$

Saturation of the machine results in a change of the $d$ - and $q$-axes inductances [16]. This change in inductance due to the magnetic saturation (5) and (6) can be modeled for carrier-signal injection using the Taylor series expansion as a function of the current, neglecting cross saturation due to the small magnitude of the carrier current

$$
\begin{aligned}
& L_{d}=L_{d o}-L_{d}^{\prime} i_{d s}^{r}-L_{d}^{\prime \prime} i_{d s}^{r 2} \\
& L_{q}=L_{q o}-L_{q}^{\prime} i_{q s}^{r}-L_{q}^{\prime \prime} i_{q s}^{r 2}
\end{aligned}
$$

where $L_{q}^{\prime}=\left(d L_{q} / d i_{q s}^{r}\right), L_{q}^{\prime \prime}=\left(d^{2} L_{q} / d i_{q s}^{r_{2}}\right), L_{d}^{\prime}=$ $\left(d L_{d} / d i_{d s}^{r}\right)$, and $L_{d}^{\prime \prime}=\left(d^{2} L_{d} / d i_{d s}^{r_{2}}\right)$

The polarity-dependent magnetic saturation (the term$\left.L_{d}^{\prime} i_{d s}^{r}\right)$ is dominant for saturation in the $d$-axis (5). Fig. 1 shows the polarity-dependent saturation in the $d$-axis for a PM synchronous motor. Considering Case 1, where the permanent magnet flux and armature current flux vector directions are aligned, the $d$-axis inductance decreases in (5) due to additional magnetic saturation. For Case 2, when the armature current flux and PM flux are $180^{\circ}$ out of phase, the $d$-axis inductance increases in (5). The $q$-axis inductance can be approximated as (6) with a polynomial where the second-order term is dominant.

While modeling the inductance change due to saturation is physically insightful, it is difficult to determine the resulting currents due to an applied high-frequency voltage (flux) as in

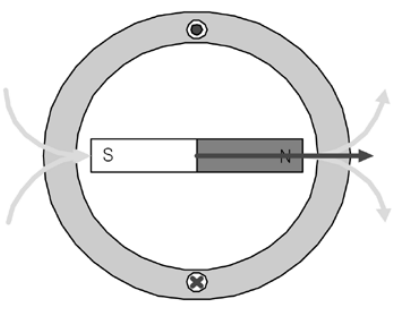

Case 1: Magnetization

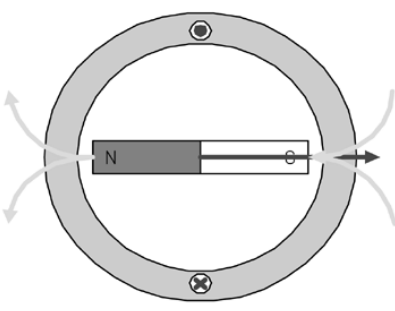

Case 2: Demagnetization
Fig. 1. Polarity-dependent magnetic saturation in the $d$ axis for PMSM.

(5) and (6). A more useful high-frequency model can be derived using the concept of reluctance. The current can be defined as a function of the reluctance multiplied by the flux. Again, a third-order Taylor series expansion for the $d$ and $q$ axes can be used to determine the complex vector current as a function of the flux in the machine (7)

$$
\begin{aligned}
i_{d q s}^{r}=\mathcal{R}_{d o} \Delta & \lambda_{d s}^{r}+\mathcal{R}_{d}^{\prime} \Delta \lambda_{d s}^{r 2} \\
& +\mathcal{R}_{d}^{\prime \prime} \Delta \lambda_{d s}^{r 3}+j\left(\mathcal{R}_{q o} \lambda_{q s}^{r}+\mathcal{R}_{q}^{\prime} \lambda_{q s}^{r 2}+\mathcal{R}_{q}^{\prime \prime} \lambda_{q s}^{r 3}\right)
\end{aligned}
$$

where

$$
\begin{aligned}
\Delta \lambda_{d s}^{r} & =\lambda_{d s}^{r}-\lambda_{p m} \\
\mathcal{R}_{d o} & =\frac{1}{L_{d o}} \\
\mathcal{R}_{d}^{\prime} & =\left.\frac{d \mathcal{R}_{d}}{d \lambda_{d s}^{r}}\right|_{\lambda_{d s}^{r}=\lambda_{p m}} \\
\mathcal{R}_{d}^{\prime \prime} & =\left.\frac{d^{2} \mathcal{R}_{d}}{d \lambda_{d s}^{r 2}}\right|_{\lambda_{d s}^{r}=\lambda_{p m}} \\
\mathcal{R}_{q o} & =\frac{1}{L_{q o}} \\
\mathcal{R}_{q}^{\prime} & =\left.\frac{d \mathcal{R}_{q}}{d \lambda_{q s}^{r}}\right|_{\lambda_{q s}^{r}=0}, \mathcal{R}_{q}^{\prime \prime}=\left.\frac{d^{2} \mathcal{R}_{q}}{d \lambda_{q s}^{r 2}}\right|_{\lambda_{q s}^{r}=0} .
\end{aligned}
$$

\section{CARrier Current for Voltage Carrier-Signal INJECTION AT STANDSTILL}

\section{A. Rotating Voltage Carrier-Signal Injection}

In order to obtain a signal with a rotor position dependency, a high-frequency voltage vector rotating at a carrier frequency $\omega_{c}(8)$ is injected into the IPM machine

$$
\boldsymbol{v}_{d q s c}^{s}=V_{c} e^{\boldsymbol{j} \theta_{c}}=V_{c}\left(\cos \omega_{c} t+\boldsymbol{j} \sin \omega_{c} t\right) .
$$

The high-frequency rotating voltage vector can be transformed to the rotor reference frame (9).

$$
\begin{aligned}
\boldsymbol{v}_{d q s c}^{r} & =\boldsymbol{v}_{d q s c}^{s} e^{-\boldsymbol{j} \theta_{r}}=V_{c} e^{\boldsymbol{j} \omega_{c} t} e^{-\boldsymbol{j} \theta_{r}} \\
& =V_{c}\left[\cos \left(\omega_{c} t-\theta_{r}\right)+\boldsymbol{j} \sin \left(\omega_{c} t-\theta_{r}\right)\right] .
\end{aligned}
$$

With high-frequency injection, the resistive drop can be neglected and the carrier stator flux (10) can be obtained by integrating the carrier-signal voltage (9) with the rotor at standstill (1) and (2)

$$
\lambda_{d q s c}^{r} \approx \int v_{d q s c}^{r} d t=\frac{V_{c}}{\omega_{c}}\left[\sin \left(\omega_{c} t-\theta_{r}\right)-j \cos \left(\omega_{c} t-\theta_{r}\right)\right] .
$$


The carrier stator flux (10) is substituted into the flux-to-current relationship (7). Thus, the interaction between the injected carrier-frequency rotating voltage vector and the saliencies produce carrier-frequency current components that contain rotor position (11)

$$
\begin{aligned}
\boldsymbol{i}_{d q s c}^{s} \approx & I_{c_{-} p 1} e^{\boldsymbol{j}\left(\omega_{c} t-\pi / 2\right)}+I_{c_{-} n 1} e^{\boldsymbol{j}\left(-\omega_{c} t+2 \theta_{r}+\phi_{1 n}\right)} \\
& +I_{c_{-} p 2} e^{\boldsymbol{j}\left(2 \omega_{c} t-\theta_{r}+\phi 2 p\right)} \\
& +I_{c_{-} n 2} e^{\boldsymbol{j}\left(-2 \omega_{c} t+3 \theta_{r}+\phi 2 n\right)}
\end{aligned}
$$

where

$$
\begin{aligned}
I_{c-p 1}= & \frac{V_{c}}{\omega_{c}} \frac{L_{q o}+L_{d o}}{2 L_{q o} L_{d o}}+\frac{3 V_{c}^{3} \mathcal{R}_{q}^{\prime \prime}}{8 \omega_{c}^{3}} \\
& -\frac{V_{c} \lambda_{p m} \mathcal{R}_{d}^{\prime}}{\omega_{c}}+\frac{\left(3 V_{c}^{3}+12 \lambda_{p m}^{2} \omega_{c}^{2} V_{c}\right) \mathcal{R}_{d}^{\prime \prime}}{8 \omega_{c}^{3}} \\
I_{c-n 1}= & \frac{V_{c}}{\omega_{c}} \frac{L_{q o}-L_{d o}}{2 L_{q o} L_{d o}}-\frac{3 V_{c}^{3} \mathcal{R}_{q}^{\prime \prime}}{8 \omega_{c}^{3}}-\frac{V_{c} \lambda_{p m} \mathcal{R}_{d}^{\prime}}{\omega_{c}} \\
& +\frac{\left(3 V_{c}^{3}+12 \lambda_{p m}^{2} \omega_{c}^{2} V_{c}\right) \mathcal{R}_{d}^{\prime \prime}}{8 \omega_{c}^{3}} \\
\phi_{1 n}= & \frac{\pi}{2} \\
I_{c-p 2, n 2}= & \frac{V_{c}^{2}}{4 \omega_{c}^{2}} \sqrt{\left(\mathcal{R}_{d}^{\prime}-3 \lambda_{p m} \mathcal{R}_{d}^{\prime \prime}\right)^{2}+\mathcal{R}_{q}^{\prime 2}} \\
\phi_{2 p, 2 n}= & \tan ^{-1}\left(-\frac{\mathcal{R}_{q}^{\prime}}{\mathcal{R}_{d}^{\prime}-3 \lambda_{p m} \mathcal{R}_{d}^{\prime \prime}}\right) .
\end{aligned}
$$

Only first- and second-order harmonic current components of carrier frequency are considered in (11), consisting of positiveand negative-sequence components. The negative sequences and second harmonic of the positive-sequence components have spatial (rotor position) information. The positive-sequence component, the first term of (11), contains no spatial information and is related to the mean value of inductance for the machine. The second harmonic of the electrical rotor position $\left(2 \theta_{r}\right)$ is contained in the second term of (11). The harmonic is primarily caused by interaction between carrier rotating signal injection and the IPM spatial saliency. The third term in (11) includes the fundamental element of the electric rotor position $\left(\theta_{r}\right)$ at the second harmonic of the positive sequence $\left(2 \omega_{c} t\right)$. This is due to saturation saliency of the $d$ axis as mentioned in Section II. The fourth term in (11) includes the third harmonic of the electrical rotor position $\left(3 \theta_{r}\right)$ at the second harmonic of the negative sequence $\left(-2 \omega_{c} t\right)$ and is also due directly to this saturation saliency.

Measured examples of the positive- and negative-sequence components in (11) are shown in Fig. 2 in a carrier current spectrum measured at standstill with a $5-\mathrm{V}$ carrier voltage at a frequency of $500 \mathrm{~Hz}$. Fig. 3 shows the first harmonic of the electrical rotor position $\left(\theta_{r}\right)$ produced by the interaction between the saturation saliencies and injected rotating carrier signal. The first harmonic of rotor position $\left(\theta_{r}\right)$ is shown in the second harmonic positive-sequence carrier $\left(2 \omega_{c} t\right)$ frame. The second harmonic of the rotor position $\left(2 \theta_{r}\right)$ in Fig. 4 is shown in the first negative carrier $\left(-\omega_{c} t\right)$ frame. The third harmonic of rotor position $\left(3 \theta_{r}\right)$ in Fig. 5 is shown in the second negative carrier $\left(-2 \omega_{c} t\right)$ frame.

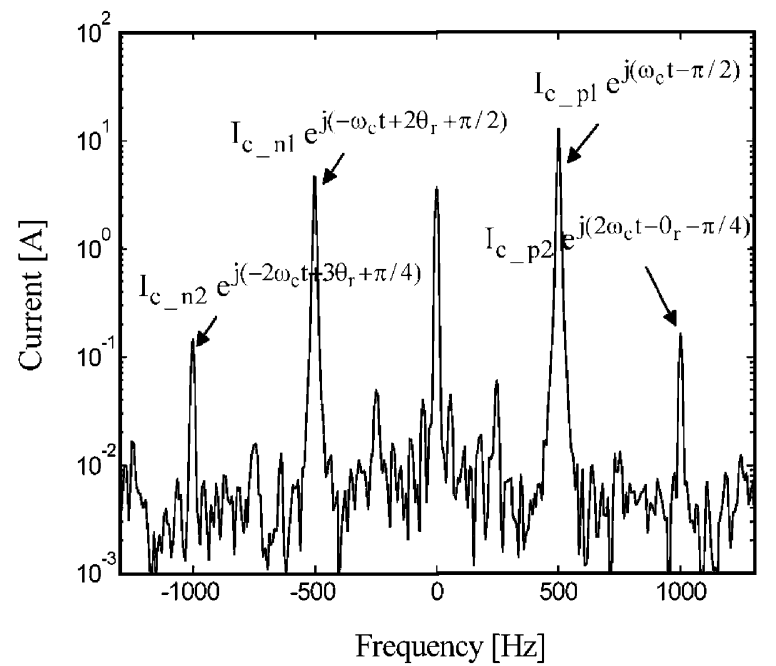

Fig. 2. Measured carrier current spectrum at standstill in the stationary reference frame.

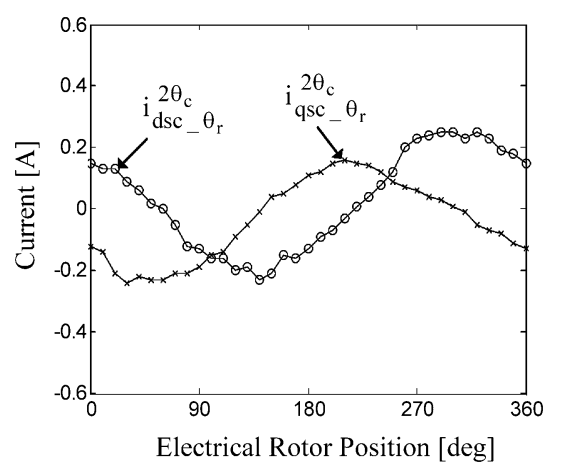

Fig. 3. Measured first harmonic of the electrical rotor position $\left(\theta_{r}\right)$ in the second positive carrier $\left(2 \omega_{c} t\right)$ reference frame.

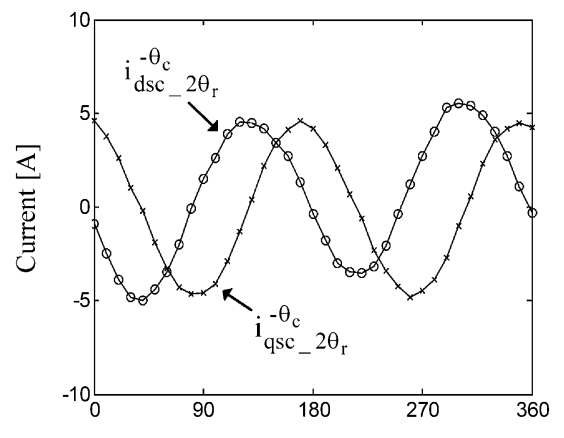

Electrical Rotor Position [deg]

Fig. 4. Measured second harmonic of the electrical rotor position $\left(2 \theta_{r}\right)$ in the first negative carrier $\left(-\omega_{c} t\right)$ reference frame.

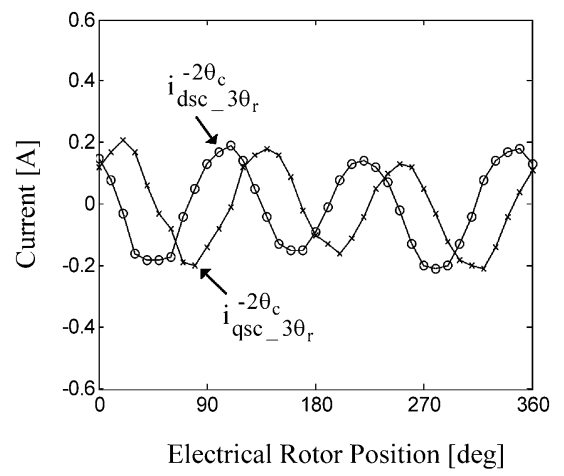

Fig. 5. Measured third harmonic of the electrical rotor position $\left(3 \theta_{r}\right)$ in the second negative carrier $\left(-2 \omega_{c} t\right)$ reference frame. 


\section{B. Pulsating Voltage Carrier-Signal Injection in the Estimated d axis}

A high-frequency pulsating voltage in the estimated $d$-axis (12) at a carrier frequency $\left(\omega_{c}\right)$ is injected into the IPM machine

$$
V_{d q s}^{\hat{r}}=V_{c} \cos \omega_{c} t
$$

The stator flux (13) due to the carrier can be obtained by integrating the carrier-signal voltage in the rotor reference frame with the rotor at standstill where the resistive drop is ignored for the high-frequency injection (1) and (2).

$$
\boldsymbol{\lambda}_{d q s c}^{r} \approx \int \boldsymbol{v}_{d q s c}^{r} d t=\frac{V_{c}}{\omega_{c}} \sin \omega_{c} t\left(\cos \theta_{\mathrm{err}}-\boldsymbol{j} \sin \theta_{\mathrm{err}}\right)
$$

where $\theta_{\text {err }}=\theta_{r}-\hat{\theta}_{r}$

The carrier stator flux (13) is substituted into the flux-to-current relationship (7). Hence, the carrier-frequency current components that contain rotor position (14) are produced by the interaction between the injected pulsating voltage carrier and the saliencies

$$
\begin{aligned}
i_{d q s}^{\hat{r}} \approx & \left(I_{c_{-} d c}+I_{c_{-} 2 \theta \mathrm{err}} e^{\boldsymbol{j} 2 \theta_{\mathrm{err}}}\right) \sin \omega_{c} t \\
+ & {\left[I_{c_{-} \theta \mathrm{err}} e^{\boldsymbol{j}\left(\theta_{\mathrm{err}}+\phi_{c \_} \theta \mathrm{err}\right.}\right) } \\
& +I_{c_{-} n \theta \mathrm{err}} e^{\boldsymbol{j}\left(-\theta_{\mathrm{err}}+\phi_{c \_n \theta \mathrm{err}}\right)} \\
& \left.+I_{c_{-} 3 \theta \mathrm{err}} e^{\boldsymbol{j}\left(3 \theta_{\mathrm{err}}+\phi_{c-3 \theta \mathrm{err}}\right)}\right] \sin ^{2} \omega_{c} t
\end{aligned}
$$

where

$$
\begin{aligned}
& I_{c_{-} d c}=\frac{V_{c}}{\omega_{c}} \frac{L_{q o}+L_{d o}}{2 L_{q o} L_{d o}}+\frac{9 V_{c}^{3} \mathcal{R}_{q}^{\prime \prime}}{32 \omega_{c}^{3}}-\frac{V_{c} \lambda_{p m} \mathcal{R}_{d}^{\prime}}{\omega_{c}} \\
& +\frac{\left(9 V_{c}^{3}+48 \lambda_{p m}^{2} \omega_{c}^{2} V_{c}\right) \mathcal{R}_{d}^{\prime \prime}}{32 \omega_{c}^{3}} \\
& I_{c \_2 \theta \mathrm{err}}=\frac{V_{c}}{\omega_{c}} \frac{L_{q o}-L_{d o}}{2 L_{q o} L_{d o}}-\frac{9 V_{c}^{3} \mathcal{R}_{q}^{\prime \prime}}{32 \omega_{c}^{3}}-\frac{V_{c} \lambda_{p m} \mathcal{R}_{d}^{\prime}}{\omega_{c}} \\
& +\frac{\left(9 V_{c}^{3}+48 \lambda_{p m}^{2} \omega_{c}^{2} V_{c}\right) \mathcal{R}_{d}^{\prime \prime}}{32 \omega_{c}^{3}} \\
& I_{c \_\theta \mathrm{err}}=\frac{V_{c}^{2}}{2 \omega_{c}^{2}} \sqrt{\left(\mathcal{R}_{d}^{\prime}-3 \lambda_{p m} \mathcal{R}_{d}^{\prime \prime}\right)^{2}+\mathcal{R}_{q}^{\prime 2}} \\
& \phi_{c_{-} \theta \mathrm{err}}=\tan ^{-1}\left(\frac{\mathcal{R}_{q}^{\prime}}{\mathcal{R}_{d}^{\prime}-3 \lambda_{p m} \mathcal{R}_{d}^{\prime \prime}}\right) \\
& I_{c \_n \theta \mathrm{err}, 3 \theta \mathrm{err}}=\frac{I_{c-\theta \mathrm{err}}}{2} \\
& \phi_{c_{-} n \theta \mathrm{err}, 3 \theta \mathrm{err}}=-\phi_{c_{-} \theta \mathrm{err}} \\
& \sin \omega_{c} t=\frac{1}{2 \boldsymbol{j}}\left(e^{\boldsymbol{j} \theta_{c}}-e^{-\boldsymbol{j} \theta_{c}}\right) \text { and }
\end{aligned}
$$

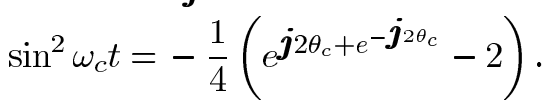

For the pulsating carrier-signal injection, the saliency image appears as an amplitude-modulated current with estimated position error in the estimated rotor frame. In contrast, the image

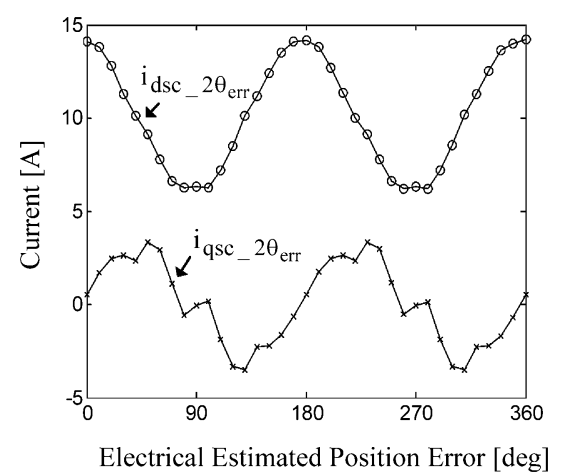

Fig. 6. Measured second harmonic components of the estimated position error through a demodulator $\left(I_{c_{-} d c}+I_{c_{-} 2 \theta \mathrm{err}} e^{\boldsymbol{j}_{2} \theta_{\text {err }}}\right)$.

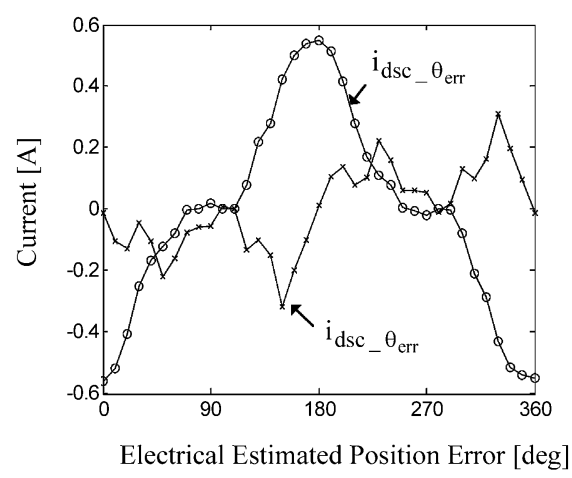

Fig. 7. Measured first harmonic components of the estimated position error through a demodulator $\left(I_{c_{-} s a t} e^{j\left(\theta_{\mathrm{err}}+\phi_{c_{-} s a t}\right)}\right)$.

appears as a carrier current with direct rotor position information in the stationary frame for the rotating carrier-signal injection. The first harmonic components of the carrier frequency $\left(\sin \omega_{c} t\right)$ in (14) are mainly produced by interaction between the pulsating signal carrier injection and the spatial saliency. Also, the second harmonic carrier-frequency current components $\left(\sin ^{2} \omega_{c} t\right)$ are generated by interaction between the carrier-signal injection and the saturation saliency.

The modulated amplitude of the second harmonic carrier-frequency components $\left(\sin ^{2} \omega_{c} t\right)$ consists of the first and third positive, and first negative harmonic of the estimated position error $\left(\theta_{\mathrm{err}}, 3 \theta_{\mathrm{err}}\right.$ and $\left.-\theta_{\mathrm{err}}\right)$. To distinguish the magnet polarity efficiently, the second harmonic carrier-frequency current components can be simplified as in (15) to a first harmonic component of the estimated position error

$$
\begin{aligned}
\boldsymbol{i}_{d q r s}^{\hat{r}} \approx\left(I_{c \_d c}+I_{c \_} 2 \theta \mathrm{err}\right. & \left.e^{\boldsymbol{j} 2 \theta_{\mathrm{err}}}\right) \sin \omega_{c} t \\
& +I_{c_{-} s a t} e^{\boldsymbol{j}\left(\theta_{\mathrm{err}}+\phi_{c \_s a t}\right)} \sin ^{2} \omega_{c} t .
\end{aligned}
$$

Fig. 6 shows the measured second harmonic components of the estimated position error $\left(I_{c_{-} d c}+I_{c_{-}} 2 \theta_{\mathrm{err}} e^{j} 2 \theta_{\text {err }}\right)$ in (15) where the first harmonic of carrier frequency $\left(\sin \omega_{c} t\right)$ is filtered off through a demodulator which will be described in Section IV.

Fig. 7 shows the measured first harmonic components of the estimated position error $\left(I_{c_{-} \text {sat }} e^{\boldsymbol{j}\left(\theta_{\mathrm{err}}+\phi_{c_{-} \text {sat }}\right)}\right)$ which was obtained through a demodulator for $\sin ^{2} \omega_{c} t$. Since the measured 


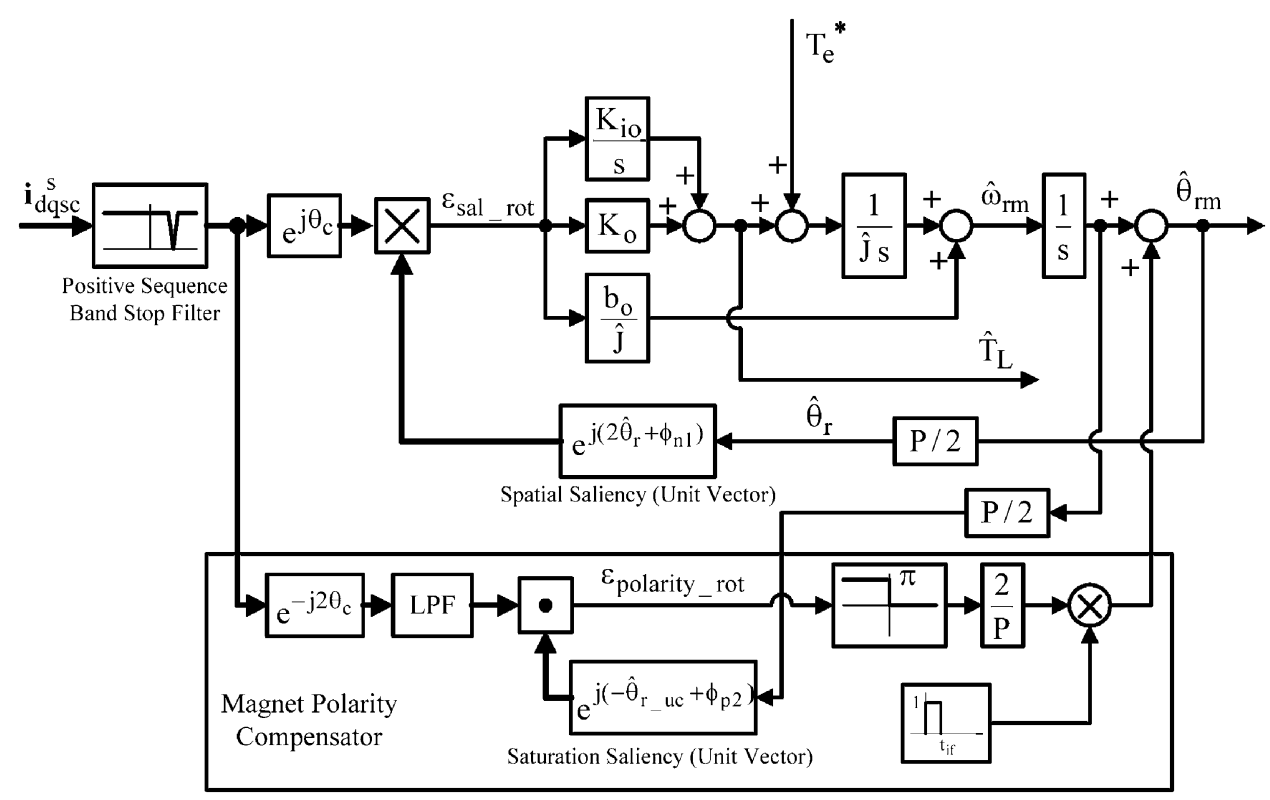

Fig. 8. Spatial saliency tracking observer with magnet polarity compensation using rotating voltage carrier-signal injection.

first harmonic component of the estimated position error $\left(\theta_{\mathrm{err}}\right)$ in (15) includes the first and third positive, and first negative harmonic of the estimated position error $\left(\theta_{\mathrm{err}}, 3 \theta_{\mathrm{err}}\right.$ and $\left.-\theta_{\mathrm{err}}\right)$ as mentioned before, it cannot be used for rotor position tracking. However, it can be used for magnet polarity compensation. The amplitude and phase of the first harmonic vector $\left(I_{C_{-} s a t}\right.$ and $\left.\phi_{c_{-} s a t}\right)$ in (15) can be estimated from the measured results of Fig. 7.

\section{INITIAL ROTOR POSITION ESTIMATION}

\section{A. Spatial Saliency Tracking Observer With Magnetic Polarity} Compensation Using Rotating Voltage Carrier-Signal Injection

A method for initial rotor position estimation is introduced using a spatial saliency tracking observer driven by the error (16) produced by the vector cross-product of the negative sequence and a unit amplitude spatial saliency vector model which is equivalent to a heterodyning process [7]

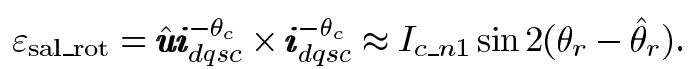

The polarity of the rotor magnet can be found by the dotproduct of the fundamental rotor position vector and a unit fundamental saliency vector model (17). When the magnet polarity error (17) is negative, the rotor position is located at a south pole from the estimated rotor position from spatial saliency tracking observer

$$
\begin{aligned}
\varepsilon_{\text {polarity_rot }} & =\hat{\boldsymbol{u}} \boldsymbol{i}_{d q s c \_\theta_{r}}^{2 \theta_{c}} \cdot \boldsymbol{i}_{d q s c \_\theta_{r}}^{2 \theta_{c}} \\
& \approx I_{c \_p 2} \cos \left(\theta_{r}-\hat{\theta}_{r_{-} u c}\right) .
\end{aligned}
$$

Fig. 8 illustrates the block diagram for the proposed initial position estimation using a spatial saliency tracking observer with magnet polarity compensation using rotating voltage carrier-signal injection. To extract only the carrier current com- ponent with the second harmonic of rotor position for rotor position estimation, positive sequence can be filtered off by a band stop filter with a low break frequency. The spatial saliency tracking observer has torque command feedforward to achieve zero-lag filtering of the position estimate [17], [18]. Since the feedforward torque command from a motion controller is zero for the initial position estimation, the tracking observer is a state filter.

\section{B. Saturation Saliency Tracking Observer Using Rotating Voltage Carrier-Signal Injection}

A second method of estimating the initial rotor position, including magnet polarity, is by using a saturation saliency tracking observer driven by the error (18) produced by the vector cross-product of the saturation saliency component with a fundamental harmonic (11) and a unit amplitude saturation saliency vector model

$$
\begin{aligned}
\varepsilon_{\text {sat_rot }} & =\hat{\boldsymbol{u}} \hat{\boldsymbol{i}}_{d q s c_{-} \theta_{r}}^{2 \theta_{c}} \times \boldsymbol{i}_{d q s c_{-} \theta_{r}}^{2 \theta_{c}} \\
& \approx I_{c_{-} p 2} \sin \left(\theta_{r}-\hat{\theta}_{r}\right) .
\end{aligned}
$$

Fig. 9 illustrates the block diagram of the proposed saturation saliency tracking observer using rotating voltage carrier-signal injection. It should be noted that rotor position tracking does not require magnet polarity detection.

\section{Spatial Saliency Tracking Observer With Magnetic Polarity Compensation Using Pulsating Voltage Carrier-Signal Injection}

Estimated position errors are shown in both positive and negative carrier frames (15) for the pulsating carrier-signal injection. Thus, the second harmonic of the estimated position error (19) fed into a rotor position tracking observer can be extracted by a demodulator which consists of positive and 


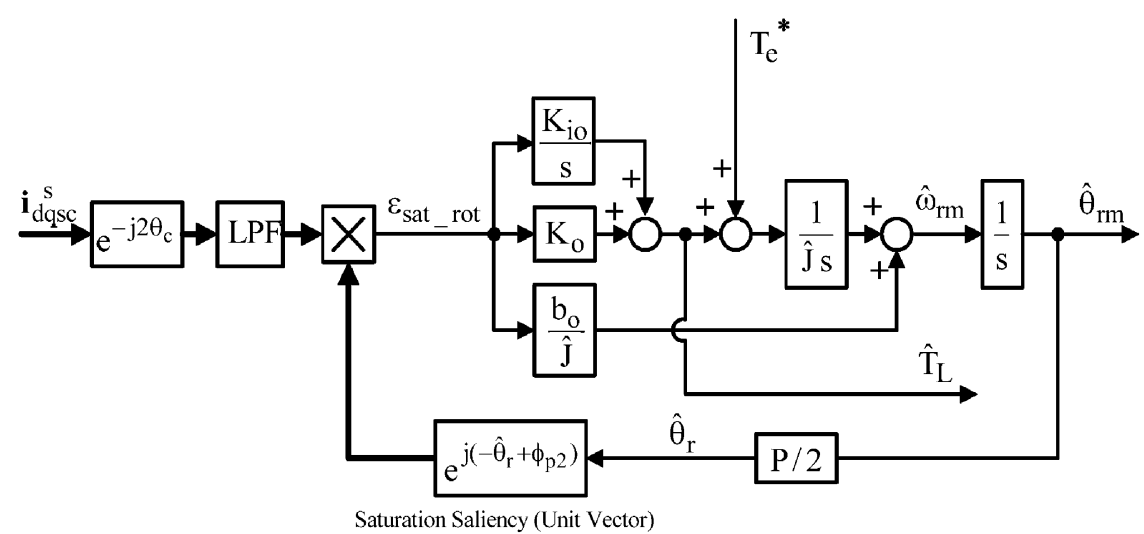

Fig. 9. Saturation saliency tracking observer using rotating voltage carrier-signal injection.

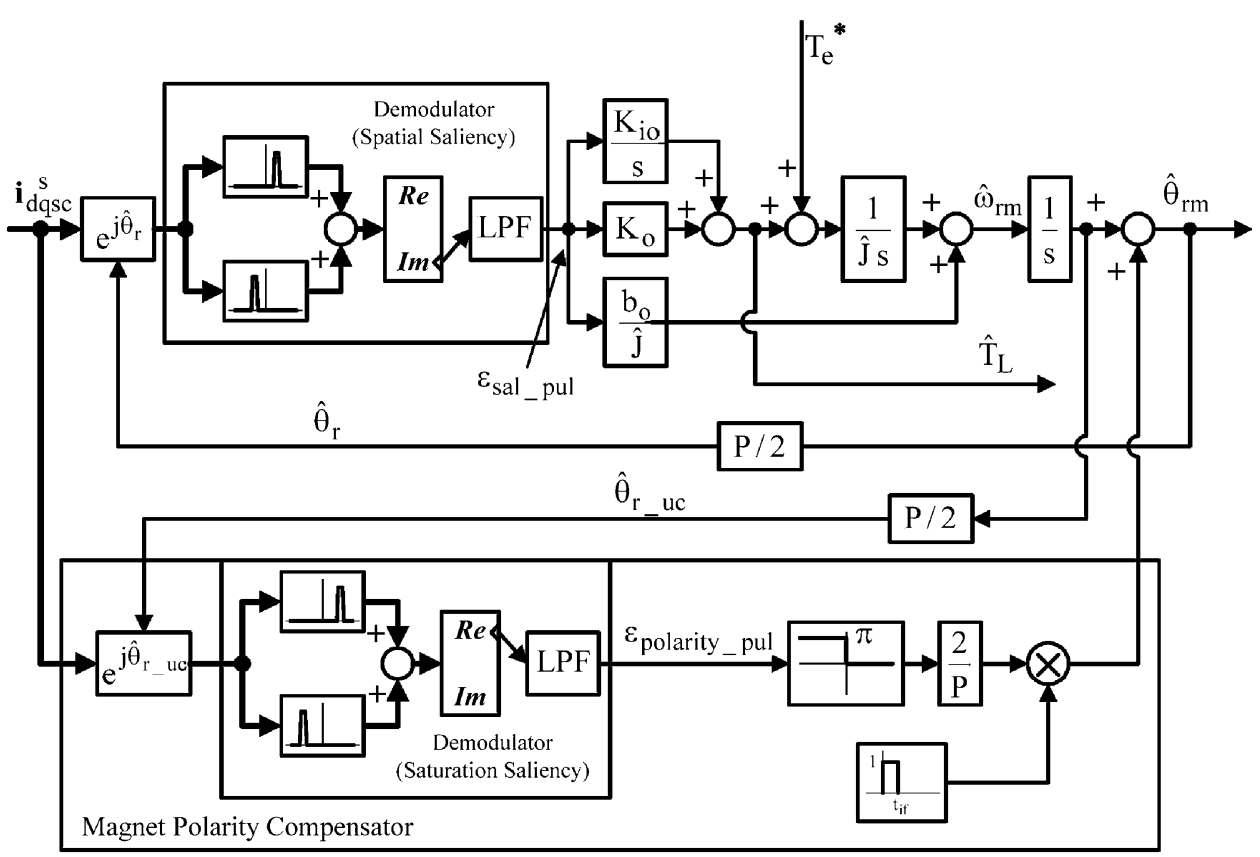

Fig. 10. Spatial saliency tracking observer with magnet polarity compensation using pulsating voltage carrier-signal injection.

negative carrier frame synchronous bandpass filter. The break frequency of the low-pass filter in the demodulator should be properly chosen to improve spatial saliency tracking and filter off the second harmonic of carrier frequency produced by the bandpass filter (19)

$$
\begin{aligned}
\varepsilon_{\text {sal_pul }}=\operatorname{Imag}\left\{\frac{\omega_{b}}{s+\omega_{b}}\right. & \\
& \left.\times\left[\left(e^{\boldsymbol{j}\left(\theta_{c}-\pi / 2\right)}+e^{\boldsymbol{j}\left(-\theta_{c}+\pi / 2\right)}\right) \boldsymbol{i}_{d q s}^{\hat{r}}\right]\right\} \\
\approx \boldsymbol{I m a g} & \left\{\frac { \omega _ { b } } { s + \omega _ { b } } \left[\left(I_{c_{-} d c}+I_{c_{-} 2 \theta \mathrm{err}} e^{\boldsymbol{j} 2 \theta \mathrm{err}}\right)\right.\right. \\
& \left.\left.\times\left(1-0.5 e^{\boldsymbol{j} 2 \theta_{c}}-0.5 e^{-\boldsymbol{j} 2 \theta_{c}}\right)\right]\right\} \\
\approx I_{c \_2 \theta_{\mathrm{err}}} & \sin 2\left(\theta_{r}-\hat{\theta}_{r}\right) .
\end{aligned}
$$

The polarity of the rotor magnet can be found from the polarity error (20) extracted in the same manner as for the demodulator (19)

$$
\begin{aligned}
& \varepsilon_{\text {polarity_pul }}=\boldsymbol{R e a l}\left\{\frac { \omega _ { b } } { s + \omega _ { b } } \left[2\left(e^{\boldsymbol{j}\left(2 \theta_{c}-\phi_{c \_ \text {sat }}+\pi\right)}\right)\right.\right. \\
& \left.\left.+e^{\boldsymbol{j}\left(-2 \theta_{c}-\phi_{c_{-} \text {sat }}+\pi\right)} \boldsymbol{i}_{d q s}^{\hat{r}}\right]\right\} \\
& \approx \boldsymbol{R e a l}\left\{\frac { \omega _ { b } } { s + \omega _ { b } } \left[I_{c_{-} \mathrm{sat}} e^{\boldsymbol{j}\left(\theta_{\mathrm{err}}+\phi_{c_{-} \mathrm{sat}}\right)}\right.\right. \\
& \left.\left.\times\left(1-e^{j 2 \theta_{c}}-e^{-j 2 \theta_{c}}+\cdots\right)\right]\right\} \\
& \approx I_{c_{-} s a t} \cos \left(\theta_{r}-\hat{\theta}_{r}\right) \text {. }
\end{aligned}
$$

Fig. 10 shows the block diagram of the proposed initial position estimation using a spatial saliency tracking observer with magnet polarity compensation using pulsating voltage carriersignal injection. 


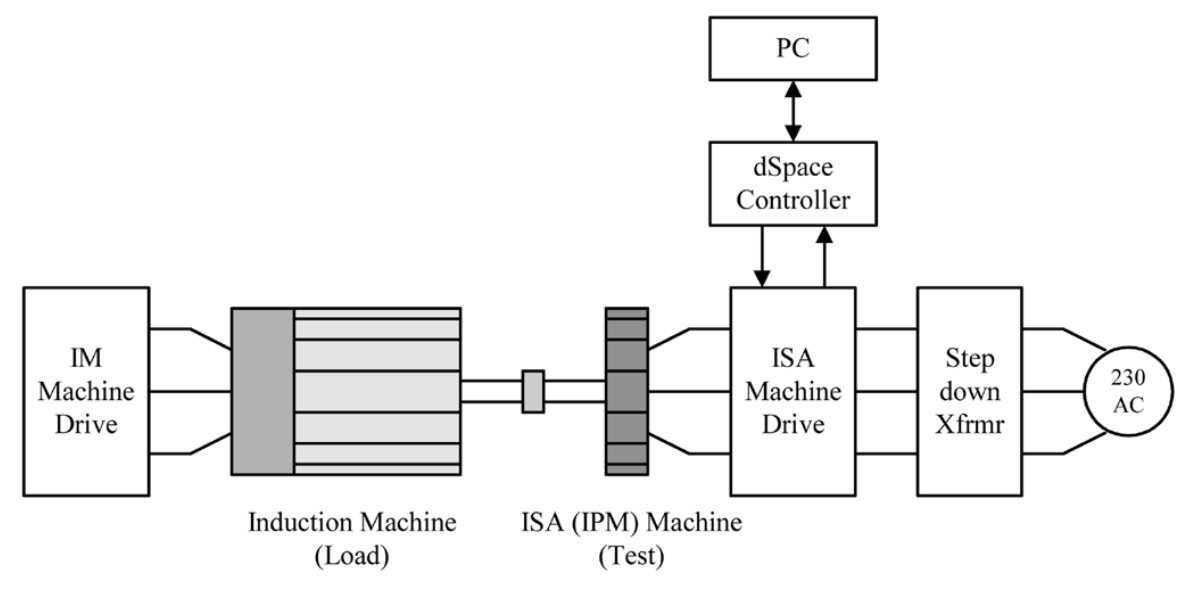

Fig. 11. Experimental test setup.

TABLE I

ISA (IPM) TEST MOTOR PARAMETERS

\begin{tabular}{c|c}
\hline Parameter & Value \\
\hline $\mathrm{R}$ & $10.3 \mathrm{~m} \Omega$ \\
$\mathrm{Lq}$ & $306 \mu \mathrm{H}$ at $10 \mathrm{~A}$ \\
$\mathrm{~L}_{\mathrm{d}}$ & $101 \mu \mathrm{H}$ at $10 \mathrm{~A}$ \\
$\lambda \mathrm{pm}$ & $0.0063 \mathrm{~Wb}$ \\
$\mathrm{~J}$ & $63.3 \times 10^{-3} \mathrm{~kg} \cdot \mathrm{m}^{2}$ \\
Poles & 12 \\
\hline
\end{tabular}

\section{EXPERIMENTAL RESULTS}

To verify the performance of the proposed initial position detection algorithms, experiments were carried out on an IPMSM integrated starter alternator (ISA). The ISA (IPM) test machine parameters are shown in Table I. Fig. 11 shows the experimental test setup which consists of an IGBT based ISA drive, an induction motor load drive, a dSpace controller, and a PC. The switching and sampling rates of the hardware system are $10 \mathrm{kHz}$. The amplitude of the injected carrier voltage for initial position estimation is $5 \mathrm{~V}$ at $500 \mathrm{~Hz}$.

Figs. 12 and 13 show the estimated initial electrical rotor position and air gap torque of the ISA at standstill for initial angles of $0^{\circ}, 90^{\circ}, 180^{\circ}$, and $270^{\circ}$. These figures show the tracking performance at standstill and the produced air-gap torque which is estimated from torque (21) with the measured current and the machine parameters in Table I

$$
\hat{T}_{e m}=\frac{3}{4} P\left[\lambda_{p m} i_{q s}^{r}+\left(L_{d}-L_{q}\right) i_{q s}^{r} i_{d s}^{r}\right] .
$$

Fig. 12 shows that the initial rotor position including polarity is rapidly estimated at standstill by the saturation saliency tracking observer of Fig. 9 or the spatial saliency with a polarity compensation of Fig. 8 using rotating voltage carrier-signal injection. The convergence time of about $10 \mathrm{~ms}$ for the spatial saliency tracking observer with a polarity compensation is faster than that of about $20 \mathrm{~ms}$ for the saturation saliency tracking observer. The relatively slow convergence for the saturation saliency tracking observer is caused by low amplitude of the second positive carrier signal $\left(2 \omega_{c} t\right)$ as shown in Fig. 3. The low amplitude has the effect on the accuracy of the estimated position. The estimated position error is respectively less than $5^{\circ}$ for the spatial saliency tracking observer and less than $20^{\circ}$ for the saturation saliency tracking observer. The machine rotor did not move due to the low magnitude of the produced air gap torque.

Fig. 13 shows the estimated initial rotor position and air-gap torque using pulsating voltage carrier-signal injection at standstill. The convergence of the initial position estimation relies on the bandwidth of the observer which is limited by undesired harmonic content. The low-pass filter was used to eliminate the second harmonic of the injected carrier frequency produced by the demodulator for the spatial saliency tracking (19). The filter can affect the convergence speed of the initial position estimation. The initial estimation has been shown to converge in about $20 \mathrm{~ms}$. The carrier voltage injection in the estimated $d$ axis produces almost zero torque at standstill in steady state. It is shown in cases 2 and 4 of Fig. 13 that relatively large torque ripple exists for initial position error of $90^{\circ}$ and $270^{\circ}$. The initial estimated position is zero with a position error of $90^{\circ}$ or $270^{\circ}$ before the start of estimation in both cases. Therefore, $q$-axis current in the rotor frame is produced until the initial position estimation converges to the rotor position without polarity detection.

The estimated results for four different initial positions: $0^{\circ}$, $90^{\circ}, 180^{\circ}$, and $270^{\circ}$, are shown in Figs. 12 and 13 . The cases are used as examples of various initial position estimation errors at the beginning of tracking where the initial conditions of the proposed initial rotor position estimators are initialized to zero. The observers will converge to the correct initial position for any selected position. The accuracy of initial position estimation is dependent on the magnitude of unmodeled spatial saliency harmonics, which, for example, might be caused by saturation.

Three observers for initial position estimation are proposed in the paper. Each observer has some application-dependent tradeoffs. A saturation saliency tracking observer using rotating voltage carrier-signal injection can be applied to both IPMSM (salient pole) and SPMSM (nonsalient pole) machines. The implementation of the saturation saliency tracking observer is simple, convergence time is very low and accuracy is quite good. The estimation accuracy and implementation 

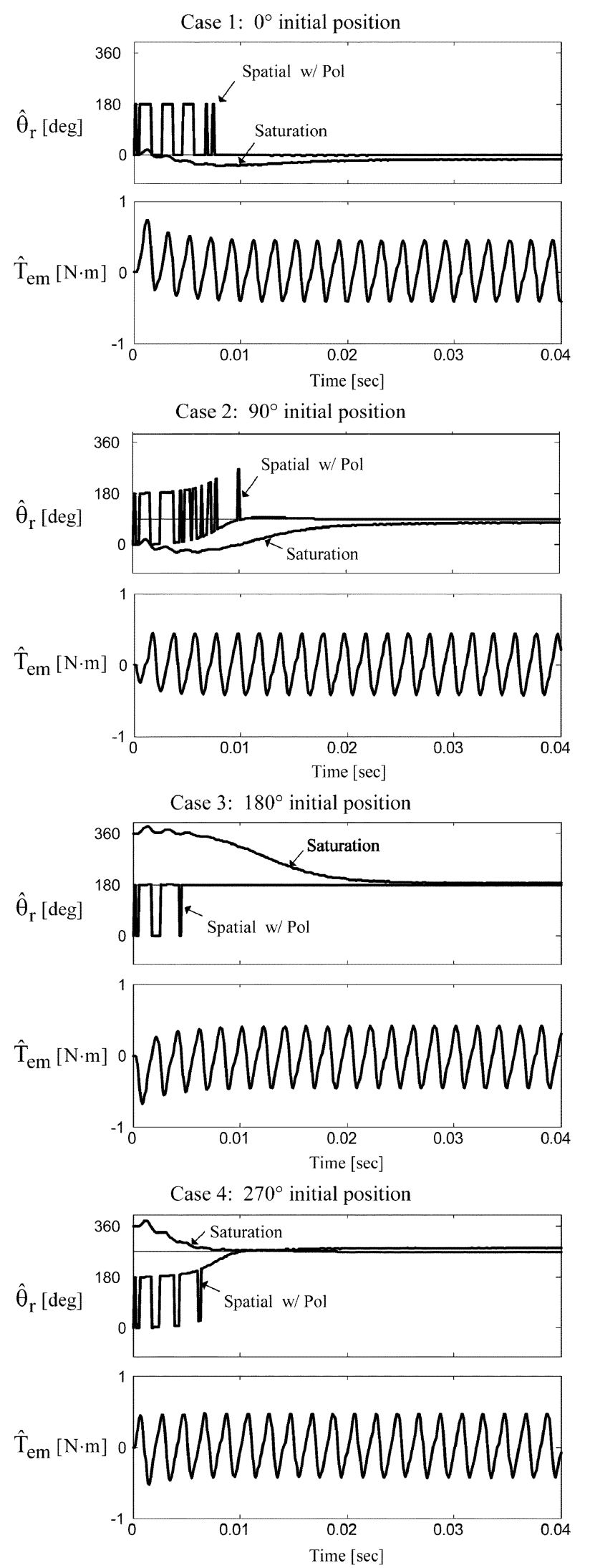

Fig. 12. Estimated initial electrical rotor position, magnet polarity, and air-gap torque using rotating voltage carrier-signal injection at standstill.

complexity of both spatial saliency tracking observers with magnet polarity compensation using rotating and pulsating
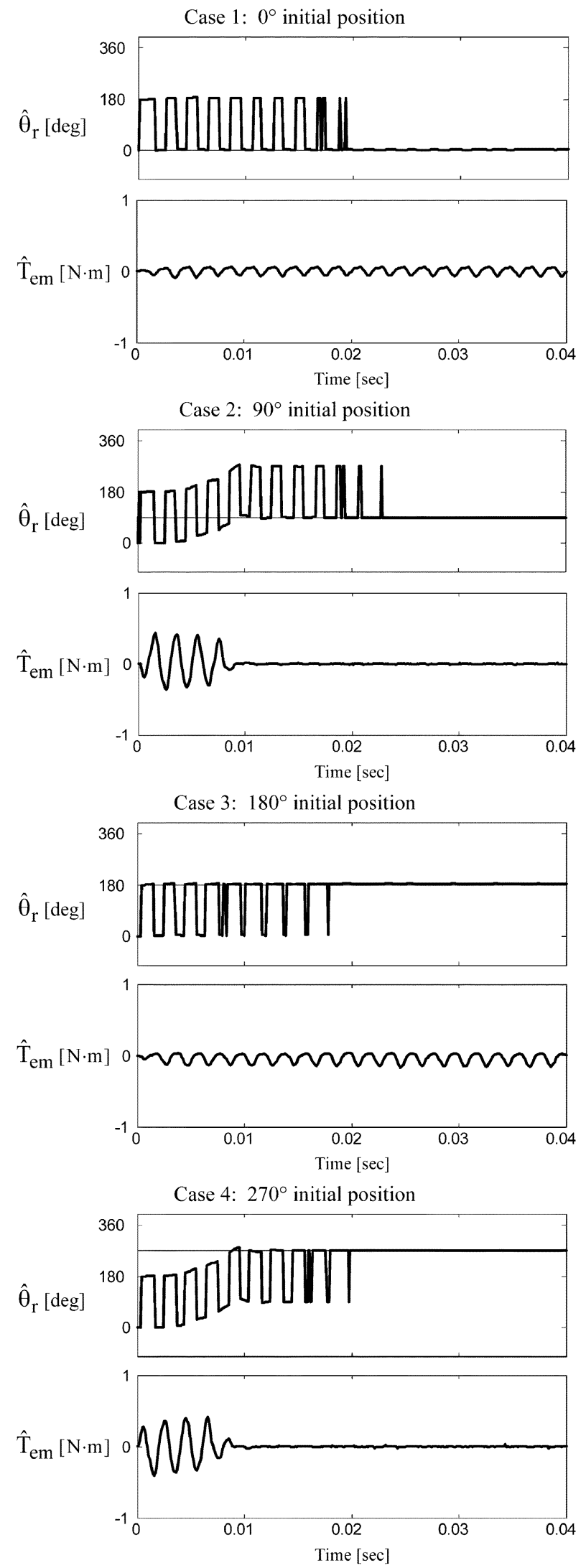

Fig. 13. Estimated initial electrical rotor position, magnet polarity, and air-gap torque using pulsating voltage carrier-signal injection at standstill.

voltage carrier-signal injection are identical overall. The spatial saliency tracking observer with magnet polarity compensation 
using rotating carrier-signal injection is more appropriate for an application with high mechanical inertia which require fast convergence. Pulsating voltage carrier-signal injection produces lower air gap torque during initial position estimation. The spatial saliency tracking observer with magnet polarity compensation using pulsating carrier-signal injection is thus more suitable for applications with low mechanical inertia. However, the convergence speed using pulsating carrier-signal injection is slower than rotating carrier-signal injection.

\section{CONCLUSION}

Three algorithms were presented for sensorless estimation of the initial position and polarity of an IPMSM. The methods converged to the correct initial position and polarity at standstill, although the convergence time depended on the method. A summary of the work and some conclusions are as follows.

- An IPMSM including magnetic saturation with carriersignal injection is presented to identify the polarization of the rotor magnets in the cavities in order to distinguish the north and south poles.

- Approaches to initial position estimation including magnet polarity have been proposed based on rotating or pulsating carrier voltage injection on spatial and saturation saliencies.

- Initial rotor position is estimated from a spatial saliency tracking estimator. Magnet polarity is identified simultaneously by using the fundamental harmonic of rotor position in the second harmonic frame of the carrier frequency due to $d$ and $q$-axis saturation saliencies for both rotating and pulsating voltage carrier injection.

- Initial position estimation is achieved by tracking the fundamental harmonic of rotor position $\left(\theta_{r}\right)$ in the second positive-sequence carrier frame $\left(2 \theta_{c}\right)$ without polarity compensation for rotating voltage carrier injection. The method can be also applied to surface permanent magnet synchronous machines.

- Robust and fast initial rotor position and polarity estimation without shaft rotation can be guaranteed by the proposed tracking estimators.

\section{REFERENCES}

[1] J. Wai and T. M. Jahns, "A new control technique for achieving wide constant power speed operation with an interior PM alternator machine," in Conf. Rec. IEEE-IAS Annu. Meeting, vol. 2, Sept./Oct. 2001, pp. 807-814.

[2] P. B. Schmidt, M. L. Gaspery, G. Ray, and A. H. Wijenayake, "Initial rotor angle detection of a nonsalient pole permanent magnet synchronous machine," in Conf. Rec. IEEE-IAS Annu. Meeting, vol. 1, 1997, pp. 459-463.

[3] S. Nakashima, Y. Inagaki, and I. Miki, "Sensorless initial rotor position estimation of surface permanent-magnet synchronous motor," IEEE Trans. Ind. Applicat., vol. 36, pp. 1598-1603, Nov./Dec. 2000.

[4] K. Tanaka, T. Yuzawa, R. Moriyama, and I. Miki, "Initial rotor position estimation for surface permanent magnet synchronous motor," in Conf. Rec. IEEE-IAS Annu. Meeting, Chicago, IL, Sept./Oct. 2001, pp. 2592-2597.

[5] T. Takeshita and N. Matsui, "Sensorless control and initial position estimation of salient-pole brushless DC motor," in Proc. Advanced Motion Control Workshop, 1996, pp. 18-23.

[6] M. Schroedl, "Sensorless control of ac machines at low speed and standstill based on the "INFORM" method," in Conf. Rec. IEEE-IAS Annu. Meeting, Oct. 6-10, 1996, pp. 270-277.
[7] P. L. Jansen, M. Corley, and R. D. Lorenz, "Flux, position, and velocity estimation in AC machines at zero and low speed via tracking of high frequency saliencies," in Proc. EPE Conf., 1995, pp. 154-160.

[8] S. Kondo, A. Takahashi, and T. Nishida, "Armature current locus-based estimation method of rotor position of permanent magnet synchronous motor without mechanical sensor," in Conf. Rec. IEEE-IAS Annu. Meeting, vol. 1, 1995, pp. 55-60.

[9] F. Philippen, "Position estimation in PM synchronous machines using single saliency-tracking, self-sensing methods," M.S. \& Diplomarbeit thesis, Univ. Wisconsin, Madison, and Tech. Univ. Aachen, Germany, 1998.

[10] A. Consoli, G. Scarcella, and A. Testa, "Sensorless control of PM synchronous motors at zero speed," in Conf. Rec. IEEE-IAS Annu. Meeting, Phoenix, AZ, Oct. 1999, pp. 1033-1040.

[11] M. Corley and R. D. Lorenz, "Rotor position and velocity estimation for a permanent magnet synchronous machine at standstill and high speeds," IEEE Trans. Ind. Applicat., vol. 34, pp. 784-789, July/Aug. 1998.

[12] T. Noguchi, K. Yamada, S. Kondo, and I. Takahashi, "Initial rotor position estimation method of sensorless PM synchronous motor with no sensitivity to armature resistance," IEEE Trans. Ind. Electron., vol. 45 , pp. 118-125, Feb. 1998

[13] D.-W. Chung, J.-K. Kang, and S. Sul, "Initial rotor position detection of PMSM at standstill without rotational transducer," in Proc. IEEE IEMDC, May 1999, pp. 785-787.

[14] J.-I. Ha, K. Ide, T. Sawa, and S. Sul, "Sensorless position control and initial position estimation of an interior permanent magnet motor," in Conf. Rec. IEEE-IAS Annu. Meeting, Chicago, IL, Sept./Oct. 2001, pp. 2607-2613.

[15] Y. Jeong, R. D. Lorenz, T. M. Jahns, and S. Sul, "Initial rotor position estimation of an interior permanent magnet synchronous machine using carrier-frequency injection methods," in Proc. IEEE IEMDC, June 2003 pp. 1218-1223.

[16] H. Kim, J. Hartwig, and R. D. Lorenz, "Using on-line parameter estimation to improve efficiency of IPM machine drives," in Proc. IEEE PESC 2002, pp. 815-820.

[17] R. D. Lorenz, "Observers and state filter in drives and power electronics," J. Elect. Eng., vol. 2, pp. 4-12, 2002.

[18] H. Kim, M. C. Harke, and R. D. Lorenz, "Sensorless control of interior permanent magnet machine drives with zero-phase lag position estimation," in Conf. Rec. IEEE-IAS Anпи. Meeting, 2002, pp. 86-91.

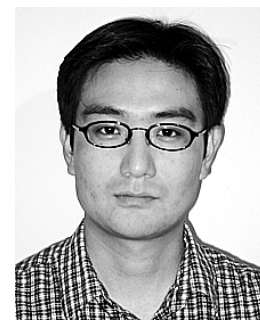

self-sensing
Hyunbae Kim (S'01) received the B.S. degree from Kunsan National University, Kunsan, Korea, in 1996 and the M.S., and Ph.D. degrees in mechanical engineering from the University of Wisconsin, Madison, in 1999 and 2004 respectively.

$\mathrm{He}$ is currently a Senior Engineer with the Living Appliances R\&D Center, Samsung Electronics, Suwon, Korea, where his research is focused on control systems and power electronics in living appliances. His research interest is high-performance permanent-magnet machine drives, including

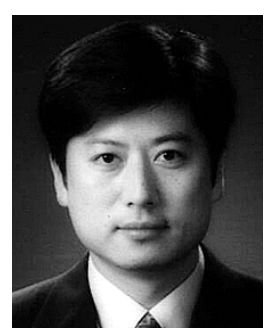

Kum-Kang Huh (S'03) was born in Seoul, Korea $\mathrm{He}$ received the B.S. and M.S. degrees in control and instrumentation engineering from Korea University, Seoul, Korea, in 1993 and 1995, respectively. He is currently working toward the Ph.D. degree in electrical engineering at the University of Wisconsin, Madison

From 1995 to 2002, he was with the Mando R\&D Center, Dukso, Korea, where he worked on the development of electric vehicle traction systems and electric power steering systems. His current research interests are in control, estimation, and diagnostics for electric machines and actuators. 


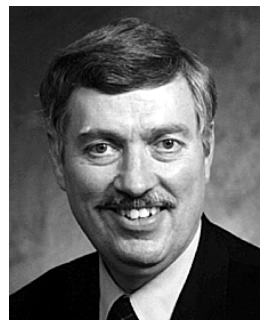

Robert D. Lorenz (S'83-M'84-SM'91-F'98) received the B.S., M.S., and Ph.D. degrees from the University of Wisconsin, Madison, and the M.B.A. degree from the University of Rochester, Rochester, NY.

Since 1984, he has been a member of the faculty of the University of Wisconsin, Madison, where he is the Mead Witter Foundation Consolidated Papers Professor of Controls Engineering in both the Department of Mechanical Engineering and the Department of Electrical and Computer Engineering. He is Co-Director of the Wisconsin Electric Machines and Power Electronics Consortium, which celebrated its 20th anniversary in 2001. It is the largest industrial research consortium on motor drives in the world. He is also the thrust leader for control and sensor integration in the Center for Power Electronic Systems, an NSF Engineering Research Center (ERC) which is a joint ERC with Virgina Polytechnic Institute and State University, Rensselaer Polytechnic Institute, University of Puerto Rico-Mayaguez, and North Carolina A\&T. From 1972 to 1982, he was a member of the research staff at the Gleason Works, Rochester, NY, working principally on high-performance drives and synchronized motion control. He was a Visiting Research Professor in the Electrical Drives Group, Catholic University of Leuven, Leuven, Belgium, in the summer of 1989 and in the Power Electronics and Electrical Drives Institute, Technical University of Aachen, Aachen, Germany, in the summers of 1987, 1991, 1995, 1997, and 1999, where he also was the SEW Eurodrive Guest Professor from September 1, 2000 until July 7, 2001. In 1969-1970, he conducted Master thesis research in adaptive control of machine tools at the Technical University of Aachen. His current research interests include sensorless electromagnetic motor/actuator technologies, real-time signal processing and estimation techniques, precision multiaxis motion control, and ac/dc drive and high-precision machine control technologies. He has authored more than 160 published technical papers and is the holder of 16 patents, with two more pending.

Dr. Lorenz was the IEEE Industry Applications Society (IAS) President for 2001, a Distinguished Lecturer of the IAS for 2000/2001, immediate past Chair of the IAS Awards Department, and past Chairman of the IAS Industrial Drives Committee, and is a member of the IAS Industrial Drives, Electric Machines, Industrial Power Converter, and Industrial Automation and Control Committees. $\mathrm{He}$ is also the current Chair of the Periodicals Committee for the IEEE Technical Activities Board. He is a member of the IEEE Sensor Council AdCom. He was awarded the 2003 IEEE IAS Outstanding Achievement award, which honors his outstanding contributions and technological developments in the application of electricity to industry. He has won 15 prize paper awards. He is a Member of the American Society of Mechanical Enginees, Instrument Society of America, and The International Society for Optical Engineers. He is a Registered Professional Engineer in the States of New York and Wisconsin.

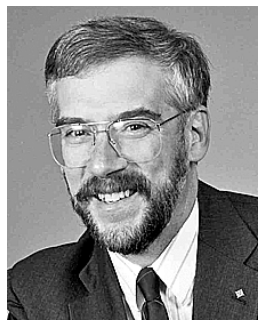

Thomas M. Jahns (S'73-M'79-SM'91-F'93) received the S.B. and S.M. degrees in 1974 and the $\mathrm{Ph} . \mathrm{D}$. degree in 1978 from Massachusetts Institute of Technology, Cambridge, all in electrical engineering.

In 1998, he joined the faculty of the University of Wisconsin, Madison, as a Professor in the Department of Electrical and Computer Engineering, where he is also an Associate Director of the Wisconsin Electric Machines and Power Electronics Consortium (WEMPEC). Prior to joining the University of Wisconsin, he was with GE Corporate Research and Development, Schenectady, NY, for 15 years, where he pursued new power electronics and motor drive technology in a variety of research and management positions. His research interests include permanent-magnet synchronous machines for a variety of applications ranging from high-performance machine tools to low-cost appliance drives. During 1996-1998, he conducted a research sabbatical at Massachusetts Institute of Technology, where he directed research activities in the area of advanced automotive electrical systems and accessories as co-director of an industry-sponsored automotive consortium.

Dr. Jahns was awarded the William E. Newell Award by the IEEE Power Electronics Society (PELS) in 1999. He has been recognized as a Distinguished Lecturer by the IEEE Industry Applications Society (IAS) during 1994-1995 and by PELS during 1998-2000. He has served as President of PELS (1995-1996) and as a Member of the IAS Executive Board between 1992-2001. He was elected Director/Delegate of IEEE Division II, serving during 2002-2003 on the IEEE Board of Directors. 